TRANSACTIONS OF THE

AMERICAN MATHEMATICAL SOCIETY

Volume 352, Number 1, Pages 243-256

S 0002-9947(99)02450-2

Article electronically published on September 21, 1999

\title{
ABSOLUTELY CONTINUOUS SPECTRUM OF PERTURBED STARK OPERATORS
}

\author{
ALEXANDER KISELEV
}

\begin{abstract}
We prove new results on the stability of the absolutely continuous spectrum for perturbed Stark operators with decaying or satisfying certain smoothness assumption perturbation. We show that the absolutely continuous spectrum of the Stark operator is stable if the perturbing potential decays at the rate $(1+x)^{-\frac{1}{3}-\epsilon}$ or if it is continuously differentiable with derivative from the Hölder space $C_{\alpha}(R)$, with any $\alpha>0$.
\end{abstract}

\section{INTRODUCTION}

In this paper, we study the stability of the absolutely continuous spectrum of one-dimensional Stark operators under various classes of perturbations. Stark Schrödinger operators describe behavior of the charged particle in the constant electric field. The absolutely continuous spectrum is a manifestation of the fact that the particle described by the operator propagates to infinity at a rather fast rate (see, e.g. [2], [12]). It is therefore interesting to describe the classes of perturbations which preserve the absolutely continuous spectrum of the Stark operators. In the first part of this work, we study perturbations of Stark operators by decaying potentials. This part is inspired by the recent work of Naboko and Pushnitski [14]. The general picture that we prove is very similar to the case of perturbations of free Schrödinger operators [9]. In accordance with physical intuition, however, the absolutely continuous spectrum is stable under stronger perturbations than in the free case.

If in the free case the short range potentials preserving purely absolutely continuous spectrum of the free operator are given by condition (on the power scale) $|q(x)| \leq C(1+|x|)^{-1-\epsilon}$, in the Stark operator case the corresponding condition reads $|q(x)| \leq C(1+|x|)^{-\frac{1}{2}-\epsilon}$. If $\epsilon$ is allowed to be zero in the above bounds, imbedded eigenvalues may occur in both cases (see, e.g. [14], [15]). Moreover, in both cases if we allow potential to decay slower by an arbitrary function growing to infinity, very rich singular spectrum, such as a dense set of eigenvalues, may occur (see [13] for the free case and [14] for the Stark case for precise formulation and proofs of these results). The first part of this work draws the parallel further, showing that the absolutely continuous spectrum of Stark operators is preserved under perturbations satisfying $|q(x)| \leq C(1+|x|)^{-\frac{1}{3}-\epsilon}$, in particular even in the regimes where a dense set of eigenvalues occurs; hence in such cases these eigenvalues are genuinely imbedded. Similar results for the free case were proven in [9],

Received by the editors April 14, 1997.

1991 Mathematics Subject Classification. Primary 34L40, 81Q10.

(C)1999 American Mathematical Society 
[10]. Our main strategy of the proof here is similar to that in [9] and [10]: we study the asymptotics of the generalized eigenfunctions and then apply Gilbert-Pearson theory [7] to derive spectral consequences.

While the main new tool we introduce in our treatment of Stark operators is the same as in the free case, namely the a.e. convergence of the Fourier-type integral operators, there are some major differences. First of all, the spectral parameter enters the final equations that we study in a different way and this makes analysis more complicated. Secondly, we employ a different method to analyze the asymptotics. Instead of Harris-Lutz asymptotic method we study appropriate Prüfer transform variables, simplifying the overall consideration.

In the second part of the work we discuss perturbations by potentials having some additional smoothness properties, but without decay. It turns out that for Stark operators the effects of decay or of additional smoothness of potential on the spectral properties are somewhat similar. It was known for a long time that if a potential perturbing Stark operator has two bounded derivatives the spectrum remains purely absolutely continuous (actually, certain growth of derivatives is also allowed, see Section 2 for details or Walter [21] for the original result). We note that the results similar to Walter's on the preservation of absolutely continuous spectrum were also obtained in [4] by applying different types of technique (Mourre method instead of studying asymptotics of solutions). On the other hand, if the perturbing potential is a sequence of derivatives of $\delta$ functions in integer points on $R$ with certain couplings, the spectrum may turn pure point [3], [5], [1]. In some sense, the $\delta^{\prime}$ interaction is the most singular and least "differentiable" among all available natural perturbations of one-dimensional Schrödinger operators [11]. Hence we have very different spectral properties on the very opposite sides of the smoothness scale. This work closes part of the gap. We improve the well-known results of Walter [21] concerning the minimal smoothness required for the preservation of the absolutely

continuous spectrum and show that in fact existence and minimal smoothness of the first derivative is sufficient to imply absolute continuity of the spectrum.

After submitting this paper, the author learned about the work of J. Sahbani [17], where, in particular, the results related to Theorem 2.1 of the present work are proven. Sahbani's results are slightly stronger than Theorem 2.1: the derivative of potential $V^{\prime}(x)$ is required to be bounded and Dini continuous in order for the absolutely continuous spectrum to be preserved. In addition, he shows that the imbedded singular spectrum in this case may only consist of isolated eigenvalues. The approach employed in [17] is an extension of conjugate operator method.

\section{DeCAying PERTurbations}

Consider a self-adjoint operator $H_{q}$ defined by the differential expression

$$
H_{q} u=-u^{\prime \prime}-x u+q(x) u
$$

on the $L^{2}(-\infty, \infty)$. Let us introduce some notation. For the function $f \in L^{2}$ we denote by $\Phi f$ its Fourier transform:

$$
\Phi f(k)=L^{2}-\lim _{N \rightarrow \infty} \int_{-N}^{N} \exp (i k x) f(x) d x .
$$


For a locally integrable function $g$ we denote by $M^{+} g$ the function

$$
M^{+} g(x)=\sup _{1>h>0} \frac{1}{2 h} \int_{0}^{h}|g(x+t)+g(x-t)| d t .
$$

We denote by $\mathcal{M}^{+} f$ the set where $M^{+} f$ is finite. By the general results on the maximal functions (see, e.g. [16]), it is easy to conclude that the complement of the set $\mathcal{M}^{+}$has Lebesgue measure zero. We will prove the following theorem:

Theorem 1.1. Suppose that the potential $q(x)$ satisfies $|q(x)| \leq C(1+|x|)^{-\frac{1}{3}-\epsilon}$, $\epsilon>0$. Then the absolutely continuous spectrum of multiplicity one fills the whole real axis. Imbedded singular spectrum may occur, but only on the complement of the set

$$
S=\frac{c}{3} \mathcal{M}^{+}\left(\Phi\left(\exp \left(2 i x^{3}-i \int_{0}^{x^{3}} q\left(c t^{\frac{2}{3}}\right) c^{-1} t^{-\frac{2}{3}} d t\right) q\left(c x^{2}\right) x^{\frac{1}{6}}\right)\right),
$$

where $c=\left(\frac{3}{2}\right)^{\frac{2}{3}}$.

We will prove Theorem 1.1 by studying the asymptotics of the solutions of the equation

$$
-u^{\prime \prime}-x u+q(x) u=\lambda u .
$$

We will then apply the subordinacy theory developed by Gilbert and Pearson [7] to derive spectral consequences. For future reference, we summarize here one of the results of the subordinacy theory for Schrödinger operators defined on the whole axis due to Gilbert [6] that we will use.

Let us call the solution $u_{1}$ of the equation (1.1) subordinate on the right if for any different solution $u_{2}$ the limit

$$
\lim _{N \rightarrow+\infty} \frac{\left\|u_{1}\right\|_{L^{2}(0, N)}}{\left\|u_{2}\right\|_{L^{2}(0, N)}}
$$

is equal to zero. Subordinacy on the left is defined similarly. The set of the energies at which there is no solution subordinate both on the right and on the left constitutes the essential support of the absolutely continuous part of the spectral measure of the Schrödinger operator. Moreover, if for every $\lambda$ from this set there exists a solution subordinate either on the right or on the left, the absolutely continuous spectrum has multiplicity one.

Since in our case the total potential grows to plus infinity on the negative semiaxis, it is clear that for every $\lambda$ there is a solution subordinate on the left and hence we need only to study the behavior of solutions on the positive semi-axis.

In studying Schrödinger operators with sufficiently smooth potentials going to $-\infty$ as $x \rightarrow \infty$ one of the useful tools is Liouville transform (see, e.g. [8] or [14]). Suppose that the equation is given by

$$
-y^{\prime \prime}+(v(x)+q(x)) y=\lambda y,
$$

where $v$ is two times differentiable and goes to $-\infty$ as $x \rightarrow \infty$ sufficiently slowly (specifically, $\int_{1}^{\xi} d x /|v(x)|^{\frac{1}{2}}$ diverges as $\xi \rightarrow \infty$ ) and $q$ is a perturbation. The 
Liouville transformation is given by

$$
\xi(x)=\int_{0}^{x} \sqrt{|v(s)|} d s, \quad \rho(\xi)=|v(x(\xi))|^{\frac{1}{4}} y(x(\xi)) .
$$

The function $\rho$ satisfies the equation

$$
-\rho^{\prime \prime}+Q(\xi) \rho=\rho,
$$

where

$$
Q(\xi)=\frac{5\left|v^{\prime}(x(\xi))\right|^{2}}{16|v(x(\xi))|^{3}}-\frac{v^{\prime \prime}(x(\xi))}{4|v(x(\xi))|^{2}}+\frac{q(x(\xi))-\lambda}{|v(x(\xi))|} .
$$

In some cases this new equation does not contain infinite potential and is simpler to deal with.

In our case $v(x)=-x$ and we bring the equation (1.1) to a convenient form using the following explicit Liouville transformation (see also [14]): $\xi=\frac{2}{3} x^{\frac{3}{2}}, \omega(\xi)=$ $x(\xi)^{\frac{1}{4}} u(x(\xi))$. Then the function $\omega$ solves the Schrödinger equation

$$
-\omega^{\prime \prime}+\left(\frac{5}{36 \xi^{2}}+\frac{-\lambda+q\left(c \xi^{\frac{2}{3}}\right)}{c \xi^{\frac{2}{3}}}\right) \omega=\omega
$$

(recall $\left.c=\left(\frac{3}{2}\right)^{\frac{2}{3}}\right)$. We introduce the short-hand notation

$$
b(\xi)=\frac{5}{36 \xi^{2}}+\frac{q\left(c \xi^{\frac{2}{3}}\right)}{c \xi^{\frac{2}{3}}}
$$

and $V(\xi, \lambda)$ for the total potential in the equation (1.3). Let us further apply Prüfer transformation to the equation for $\omega$, setting for each $\lambda$

$$
\begin{aligned}
\omega(\xi, \lambda) & =R(\xi, \lambda) \sin (\theta(\xi, \lambda)), \\
\omega^{\prime}(\xi, \lambda) & =R(\xi, \lambda) \cos (\theta(\xi, \lambda)) .
\end{aligned}
$$

The equations for $R$ and $\theta$ are as follows:

$$
\begin{gathered}
(\log R(\xi, \lambda))^{\prime}=\frac{1}{2} V(\xi, \lambda) \sin (2 \theta(\xi, \lambda)) \\
\theta^{\prime}(\xi, \lambda)=1-\frac{1}{2} V(\xi, \lambda)(1-\cos (2 \theta(\xi, \lambda))) .
\end{gathered}
$$

Our goal now is to study the asymptotics of solutions of (1.3). In particular, we would like to establish convergence of the integral

$$
\int_{0}^{N}\left(\frac{5}{36 \xi^{2}}+\frac{-\lambda+q\left(c \xi^{\frac{2}{3}}\right)}{c \xi^{\frac{2}{3}}}\right) \sin (2 \theta(\xi, \lambda)) d \xi
$$

as $N \rightarrow \infty$ for a.e. $\lambda$. This goal is motivated by the following

Lemma 1.2. Suppose that for some value of $\lambda$ the integral (1.6) converges for all initial data $\theta(0, \lambda)$. Then for this value of $\lambda$, there is no subordinate solution of the original equation (1.1). Moreover, if the integral (1.6) converges for a.e. $\lambda$, the absolutely continuous part of the spectral measure of the operator $H_{q}$ fills the whole real axis. 
Proof. If for a given value of $\lambda$ the integral (1.6) converges for every initial condition of $\theta$, it follows from (1.4) and (1.5) that all solutions of the equation (1.3) are bounded and moreover there are two linearly independent solutions with the following asymptotics as $\xi \rightarrow+\infty$ :

$$
\begin{aligned}
& \omega_{1}(\xi, \lambda)=\sin \left(\xi+r_{1}(\xi, \lambda)\right)(1+o(1)), \\
& \omega_{2}(\xi, \lambda)=\cos \left(\xi+r_{2}(\xi, \lambda)\right)(1+o(1)),
\end{aligned}
$$

where $r_{i}^{\prime}(\xi, \lambda) \leq C(1+\xi)^{-\frac{2}{3}}$. Going back to the original equation (1.1), we infer that for a.e. $\lambda$ this equation has only solutions $u_{\alpha}(x, \lambda)$ (where $\alpha$ is some parametrization of solutions, say by boundary condition at zero) with the following asymptotical behavior as $x \rightarrow \infty$ :

$$
u_{\alpha}(x, \lambda)=x^{-\frac{1}{4}} \sin \left(\frac{2}{3} x^{\frac{3}{2}}+f_{\alpha}(x, \lambda)\right)(1+o(1)),
$$

where $\left|f_{\alpha}^{\prime}(x, \lambda)\right| \leq C(1+x)^{-\frac{1}{2}}$ uniformly over $\alpha$. For any $u_{\alpha}$ we then find

$$
\begin{gathered}
\int_{0}^{N}\left|u_{\alpha}(x)\right|^{2} d x=\int_{0}^{N} x^{-\frac{1}{2}}\left(\sin \left(\frac{2}{3} x^{\frac{3}{2}}+f_{\alpha}(x)\right)^{2} d x(1+o(1))\right. \\
=\left(N^{\frac{1}{2}}-\frac{1}{2} \int_{0}^{N} x^{-\frac{1}{2}} \cos \left(\frac{4}{3} x^{\frac{3}{2}}+2 f_{\alpha}(x, \lambda)\right) d x\right)(1+o(1))=N^{\frac{1}{2}}(1+o(1)) .
\end{gathered}
$$

The last equality follows from integrating by parts the integral in the previous expression. Hence, we obtain that for a.e. $\lambda$ there are no subordinate solutions: as $N \rightarrow \infty$, the $L^{2}$ norm grows at the same rate for all solutions. The last claim of the lemma follows by direct application of subordinacy theory.

Remark. 1. Note that in particular we obtained that for a.e. $\lambda$ all solutions of the equation (1.1) are bounded (and even power-decaying). However the results deriving absolute continuity of the spectrum from the boundedness of solutions (see, e.g., [20], [19]) are not applicable here because the potential goes to $-\infty$ on $R^{+}$. Instead, one has to directly apply Gilbert-Pearson theory.

2. Note that while for a.e. $\lambda$ there is no subordinate solution of equation (1.1) and hence the absolutely continuous spectrum fills the whole axis, there may be a complementary set of measure zero where the singular part of the spectral measure might be supported. As examples show [14], the imbedded spectrum may even be dense. The a.e. convergence of Fourier type integral operators allows us to prove a.e. convergence of the integral (1.6) while permitting exceptional measure zero set where convergence may fail.

We begin analyzing the integral (1.6) with the following simple

Lemma 1.3. Suppose that the function $h(\xi)$ satisfies

$$
h(\xi)=\xi+g(\xi)
$$

where $\left|g^{\prime}(\xi)\right| \leq C \xi^{-\frac{2}{3}}$. Then the integrals

$$
\int_{1}^{N} \xi^{-\frac{2}{3}} \exp ( \pm i h(\xi)) d \xi
$$


converge and, moreover,

$$
\int_{N}^{\infty} \xi^{-\frac{2}{3}} \exp ( \pm i h(\xi)) d \xi=O\left(N^{-\frac{1}{3}}\right) .
$$

Proof.

$$
\begin{gathered}
\int_{1}^{N} \xi^{-\frac{2}{3}} \exp ( \pm i h(\xi)) d \xi=N^{-\frac{2}{3}} \int_{1}^{N} \exp ( \pm i h(\xi)) d \xi \\
+\frac{2}{3} \int_{1}^{N} \xi^{-\frac{5}{3}} \int_{1}^{\xi} \exp ( \pm i h(\eta)) d \eta d \xi .
\end{gathered}
$$

Pick $\xi_{0}$ so that for $\xi>\xi_{0},\left|g^{\prime}(\xi)\right|<\frac{1}{2}$ (we can do it by the assumption on $g$ ). Then

$$
\int_{\xi_{0}}^{N} \exp ( \pm i h(\xi)) d \xi=\int_{h\left(\xi_{0}\right)}^{h(N)} \exp ( \pm i h) \frac{1}{1+g^{\prime}(\xi(h))} d h,
$$

where $\left.\mid g^{\prime}(\xi(h))\right) \mid \leq C_{1} h^{-\frac{2}{3}}$. Expanding the fraction in the last integral, we find that

$$
\int_{\xi_{0}}^{N} \exp ( \pm i h(\xi)) d \xi=O\left(N^{\frac{1}{3}}\right) .
$$

Hence we see that the integral in question converges. It is also straightforward to establish the last estimate of the lemma.

From Lemma 1.3 and (1.5) the integrals

$$
\int_{0}^{N} \xi^{-\frac{2}{3}} \exp ( \pm 2 i \theta(\xi, \lambda)) d \xi
$$

converge for every value of $\lambda$. Therefore, we see that to establish convergence of the integral (1.6) it suffices to study the integral

$$
\int_{1}^{N}\left(\frac{5}{36 \xi^{2}}-q\left(c \xi^{\frac{2}{3}}\right)(c \xi)^{-\frac{2}{3}}\right) \sin 2 \theta(\xi, \lambda) d \xi .
$$

(Of course, for the fast decaying term the convergence issue is trivial.)

We will need two lemmata on the a.e. convergence of Fourier-type integrals. The first lemma we need is Lemma 1.3 from [9]. We refer to that paper for a proof.

Lemma 1.4. Consider the function $f \in L^{2}(R)$. Then for every $\lambda_{0} \in \mathcal{M}^{+}(\Phi(f))$ we have

$$
\int_{-N}^{N} f(x) \exp \left(i \lambda_{0} x\right) d x=O(\log N)
$$

The second lemma is a variation of Lemma 1.4 from [9]. We provide here the statement and a sketch of the proof for the sake of completeness. 
Lemma 1.5. Suppose that a function $f(x)$ satisfies $|f(x)| \leq C(1+|x|)^{-\alpha}$, where $\alpha>\frac{1}{2}$. Then for a.e. $\lambda$ the integral

$$
\int_{0}^{N} \exp (i \lambda x) f(x) d x
$$

converges as $x \rightarrow \infty$ and moreover for every $\delta>0$

$$
\int_{N}^{\infty} \exp (i \lambda x) f(x) d x=o\left(N^{-\alpha+\frac{1}{2}+\delta}\right)
$$

for a.e. $\lambda$.

Explicitly, we can say that (1.8) holds for every $\lambda \in \mathcal{M}^{+}\left(\Phi\left(f(x) x^{\alpha-\frac{1}{2}-\epsilon}\right)\right)$ where $\epsilon<\delta$.

Proof. Consider an a priori estimate

$$
\begin{gathered}
\int_{N}^{\infty} \exp (i \lambda x) x^{\alpha-\frac{1}{2}-\epsilon} f(x) x^{-\alpha+\frac{1}{2}+\epsilon} d x=N^{-\alpha+\frac{1}{2}+\epsilon} \int_{0}^{N} \exp (i \lambda x)\left(f(x) x^{\alpha-\frac{1}{2}-\epsilon}\right) d x \\
+\left(\frac{1}{2}-\alpha+\epsilon\right) \int_{N}^{\infty} x^{-\alpha-\frac{1}{2}+\epsilon}\left(\int_{0}^{x} \exp (i \lambda y) f(y) y^{\alpha-\frac{1}{2}-\epsilon} d y\right) d x
\end{gathered}
$$

By Lemma 1.4, for every $\lambda \in \mathcal{M}^{+}\left(\Phi\left(f(x) x^{\alpha-\frac{1}{2}-\epsilon}\right)\right)$ we have an estimate

$$
\int_{0}^{N} \exp (i \lambda y) f(y) y^{\alpha-\frac{1}{2}-\epsilon} d y=O(\log N)
$$

Therefore for such $\lambda$ we obtain

$$
\int_{N}^{\infty} \exp (i \lambda x) f(x) d x=O\left(N^{-\alpha+\frac{1}{2}+\epsilon} \log N\right)=o\left(N^{-\alpha+\frac{1}{2}+\delta}\right)
$$

since $\delta>\epsilon$. The lemma is proven.

Remark. We note that by Zygmund's theorem [22] the Fourier integral converges a.e. for every $f \in L^{p}, 1 \leq p<2$. One can avoid using Lemma 1.4 by directly applying this theorem in the proof of Lemma 1.5. However this approach is less direct and also does not provide any explicit information about the convergence set.

Corollary 1.6. Suppose that the function $f(\xi)$ satisfies $|f(\xi)| \leq C(1+|\xi|)^{-\alpha-\epsilon}$, where $\alpha \geq \frac{5}{6}$ and $\epsilon$ is an arbitrarily small positive number. Then for a.e. $\lambda$ the integral

$$
\int_{0}^{N} f(\xi) \exp \left(i \lambda \xi^{\frac{1}{3}}\right) d \xi
$$

converges as $N \rightarrow \infty$. Moreover, for every $\lambda \in \mathcal{M}^{+}\left(\Phi\left(f\left(\xi^{3}\right) \xi^{3 \alpha-\frac{1}{2}}\right)\right)$ we have

$$
\left|\int_{N}^{\infty} f(\xi) \exp \left(i \lambda \xi^{\frac{1}{3}}\right) d \xi\right| \leq C N^{-\alpha+\frac{5}{6}} \text {. }
$$


Proof. The change of variables $y=\xi^{\frac{1}{3}}$ transforms the integral into the form

$$
3 \int_{0}^{N^{\frac{1}{3}}} f\left(y^{3}\right) y^{2} \exp (i \lambda y) d y
$$

Note that

$$
\left|f\left(y^{3}\right) y^{2}\right| \leq C(1+|y|)^{-3 \alpha-3 \epsilon+2}
$$

by assumption. Applying Lemma 1.5 (and remembering to take into account the lower limit) we obtain the statements of the corollary.

We prove the final lemma we need for the proof of the theorem. Let us introduce some short-hand notation:

$$
\begin{gathered}
\gamma(x, \lambda)=2 \theta(x, \lambda)-2 x+c^{-1} \int_{0}^{x}\left(q\left(c \xi^{\frac{2}{3}}\right) \xi^{-\frac{2}{3}}-\lambda \xi^{-\frac{2}{3}}\right) d \xi+\lambda c^{-1} \int_{0}^{x} \xi^{-\frac{2}{3}} \cos 2 \theta(\xi, \lambda) d \xi ; \\
\sigma(x, \lambda)=2 \theta(x, \lambda)-\gamma(x, \lambda) .
\end{gathered}
$$

From the equation (1.5) we find

$$
\gamma^{\prime}(x, \lambda)=b(x) \cos (\gamma(x, \lambda)+\sigma(x, \lambda))-\frac{5}{36 x^{2}} .
$$

Lemma 1.7. Suppose that the potential $q(x)$ satisfies $|q(x)| \leq C(1+|x|)^{-\frac{1}{3}-\epsilon}$. Then the integrals

$$
\int_{0}^{N} b(\xi) \exp ( \pm i \sigma(\xi, \lambda)) d \xi
$$

converge as $N \rightarrow \infty$ for a.e. $\lambda$ and moreover for a.e. $\lambda$

$$
\int_{N}^{\infty} b(\xi) \exp ( \pm i \sigma(\xi, \lambda)) d \xi=O\left(N^{-\frac{1}{18}}\right) .
$$

Proof. We will consider the case of the plus sign; the other case is analogous. By Lemma 1.3 and the definitions of $b(\xi)$ and $\sigma(\xi, \lambda)$, it is clear that it suffices to investigate the convergence of the following integral

$$
\int_{0}^{N} q\left(c \xi^{\frac{2}{3}}\right) \xi^{-\frac{2}{3}} \exp \left(2 i \xi-i c^{-1} \int_{0}^{\xi} q\left(c \eta^{\frac{2}{3}}\right) \eta^{-\frac{2}{3}} d \eta+3 c^{-1} \lambda i \xi^{\frac{1}{3}}+i c(\lambda)+i \xi^{-\frac{1}{3}} h(\lambda, \xi)\right),
$$

where the function $h(\xi, \lambda)$ is bounded for every $\lambda$. We used Lemma 1.3 to replace

$$
\lambda c^{-1} \int_{0}^{\xi} \eta^{-\frac{2}{3}} \cos (2 \theta(\eta, \lambda)) d \eta
$$

by

$$
c(\lambda)+\xi^{-\frac{1}{3}} h(\lambda, \xi)
$$

in the exponent in (1.11). Let us denote

$$
\tilde{q}(\xi)=q\left(c \xi^{\frac{2}{3}}\right) \xi^{-\frac{2}{3}} \exp \left(2 i \xi-i \int_{0}^{\xi} q\left(c \eta^{\frac{2}{3}}\right) c^{-1} \eta^{-\frac{2}{3}} d \eta\right) .
$$


We can rewrite the integral (1.11) as

$$
\int_{0}^{N} \tilde{q}(\xi) \exp \left(3 i \lambda c^{-1} \xi^{\frac{1}{3}}-i \xi^{-\frac{1}{3}} h(\lambda, \xi)\right) d \xi
$$

(we omit the constant factor $\exp (i c(\lambda))$ ). Note that as $\xi \rightarrow \infty$, we have

$$
\exp \left(i \xi^{-\frac{1}{3}} h(\lambda, \xi)\right)=1+\xi^{-\frac{1}{3}} \tilde{h}(\lambda, \xi)
$$

where $\tilde{h}$ is bounded in $\xi$ for every $\lambda$. Hence we can write the integral (1.11) as a sum of two integrals

$$
\int_{0}^{N} \tilde{q}(\xi) \exp \left(3 i \lambda c^{-1} \xi^{\frac{1}{3}}\right) d \xi+\int_{0}^{N} \tilde{q}(\xi) \xi^{-\frac{1}{3}} \tilde{h}(\xi, \lambda) \exp \left(3 i \lambda c^{-1} \xi\right) d \xi .
$$

Note that by assumption on the decay of $q$ and (1.12) we have

$$
|\tilde{q}(\xi)| \leq C \xi^{-\frac{8}{9}-\frac{2}{3} \epsilon} .
$$

Therefore, the second integral converges for every $\lambda$ because the integrand is absolutely integrable. It is also easy to estimate the rate of convergence for the second integral:

$$
\left|\int_{N}^{\infty} \tilde{q}(\xi) \xi^{-\frac{1}{3}} \tilde{h}(\xi, \lambda) \exp \left(3 i \lambda c^{-1} \xi\right) d \xi\right| \leq C(\lambda) N^{-\frac{2}{9}}
$$

for every $\lambda$ because of the assumption on decay of $q$. To study the behavior of the first integral, we note that because of the estimate (1.13) we can apply Corollary 1.6. We obtain that for a.e. $\lambda$ the first integral converges, and, moreover, for a.e. $\lambda$ (explicitly, for every $\lambda \in \frac{c}{3} \mathcal{M}^{+}\left(\Phi\left(\tilde{q}\left(\xi^{3}\right) \xi^{\frac{13}{6}}\right)\right)$ the estimate on the rate of convergence is given by

$$
\int_{N}^{\infty} \tilde{q}(\xi) \exp \left(3 i \lambda c^{-1} \xi^{\frac{1}{3}}\right) d \xi \leq C(\lambda) N^{-\frac{1}{18}}
$$

Combining the two estimates, we see that the lemma is proven.

We are now in a position to complete the proof of the theorem. The main idea is to take advantage of certain symmetry of the equations (1.4), (1.5) to estimate (1.6).

Proof of Theorem 1.1. As we already remarked, to complete the proof we only have to study the a.e. convergence of the integral (1.7). Using (1.9) we rewrite this integral as

$$
\int_{1}^{\infty} b(\xi) \sin (\gamma(\xi, \lambda)+\sigma(\xi, \lambda)) d \xi
$$

We will integrate by parts two times, differentiating the terms which contain $\gamma(\xi, \lambda)$. We omit the arguments of $\gamma$ and $\sigma$ in the following computations. We also omit in all following computations the short-range term $-5 / 36 x^{2}$ from (9). The part of the integrals corresponding to this term converges trivially. 


$$
\begin{gathered}
\int_{1}^{N} b(\xi) \sin (\gamma+\sigma) d \xi=\int_{1}^{N} b(\xi)(\sin \gamma \cos \sigma+\sin \sigma \cos \gamma) d \xi \\
=-\left.\left(\cos \gamma \int_{\xi}^{\infty} b(\eta) \sin \sigma d \eta+\sin \gamma \int_{\xi}^{\infty} b(\eta) \cos \sigma d \eta\right)\right|_{1} ^{N} \\
+\int_{1}^{N} b(\xi) \cos (\gamma+\sigma)\left(\cos \gamma \int_{\xi}^{\infty} b(\eta) \cos \sigma d \eta-\sin \gamma \int_{\xi}^{\infty} b(\eta) \sin \sigma d \eta\right) d \xi
\end{gathered}
$$

We remark that by Lemma 1.7 the conditional integrals are well-defined for a.e. $\lambda$. Given that, we see that for a.e. $\lambda$ the off-diagonal terms stay finite. We rearrange the integral term and write it as follows:

$$
\begin{aligned}
& \int_{1}^{N}(\sin \gamma)^{2}\left(b(\xi) \sin \sigma \int_{\xi}^{\infty} b(\eta) \sin \sigma d \eta\right) d \xi \\
-\int_{1}^{N}(\sin \gamma \cos \gamma) & \left(b(\xi) \cos \sigma \int_{\xi}^{\infty} b(\eta) \sin \sigma d \eta+b(\xi) \sin \sigma \int_{\xi}^{\infty} b(\eta) \cos \sigma d \eta\right) d \xi \\
+ & \int_{1}^{N}(\cos \gamma)^{2}\left(b(\xi) \cos \sigma \int_{\xi}^{\infty} b(\eta) \cos \sigma d \eta\right) d \xi .
\end{aligned}
$$

Integrating by parts (terms in brackets being integrated), we again obtain offdiagonal terms which are a.e. $\lambda$ finite and the following integral term:

$$
\begin{gathered}
\int_{1}^{N} \sin (2 \gamma) b(\xi) \cos (\gamma+\sigma)\left(\left(\int_{\xi}^{\infty} b(\eta) \sin \sigma d \eta\right)^{2}-\left(\int_{\xi}^{\infty} b(\eta) \cos \sigma d \eta\right)\right) d \xi \\
-\int_{1}^{N} \cos (2 \gamma) b(\xi) \cos (\gamma+\sigma)\left(\int_{\xi}^{\infty} b(\eta) \sin \sigma d \eta\right)\left(\int_{\xi}^{\infty} b(\eta) \cos \sigma d \eta\right) d \xi .
\end{gathered}
$$

Hence, the integral (1.7) and therefore (1.6) is convergent if

$$
b(\xi)\left(\int_{\xi}^{\infty} b(\eta) \exp ( \pm i \sigma(\eta, \lambda)) d \eta\right)^{2} \in L^{1}(0, \infty) .
$$

Because of the assumption on the decay of the $q$, definition of $b(\xi)$, and Lemma 1.7, we find that for a.e. $\lambda \in R$ the expression above is bounded above by

$$
C(\lambda) \xi^{-\frac{2}{3}} \xi^{-\frac{2}{9}-\frac{\epsilon}{2}} \xi^{-\frac{1}{9}} \leq C(\lambda) \xi^{-1-\frac{\epsilon}{2}}
$$

and hence is absolutely integrable for a.e. $\lambda$. This proves the convergence of the integral (1.6) for a.e. $\lambda$. Since from the proof of Lemma 1.7 follows that the estimate (1.10) holds for every $\lambda \in \frac{c}{3} \mathcal{M}^{+}\left(\Phi\left(\tilde{q}\left(\xi^{3}\right)\right) \xi^{-\frac{13}{6}}\right)$, we also obtain an explicit set to 
which the singular part of the spectral measure gives zero weight, as stated in the theorem.

\section{The CASE OF SUfFiciently SMOoth Potential}

In this section, we take up the study of an alternative kind of conditions implying the absolute continuity of the spectrum of Stark operators. We begin with the remark that if the perturbation $q(x)$ is twice differentiable, $\left|q^{\prime}(x)\right| \leq C(1+|x|)$ and $\left|q^{\prime \prime}(x)\right| \leq C(1+|x|)^{\alpha}, \alpha<\frac{1}{2}$, we can apply the Liouville transformation directly to the whole potential $-x+q(x)$, obtaining the equation (2) with absolutely integrable potential $Q$. Then by Lemma 1.2 , for every $\lambda$ there is no subordinate solution of the original equation and hence the spectrum is purely absolutely continuous. The situation is more subtle if $q$ does not have two derivatives and hence we cannot apply Liouville transformation directly to the whole potential $-x+q(x)$. Our goal is to show the following improvement of results proven in [21], [4]:

Theorem 2.1. Suppose that the perturbation $q(x)$ is bounded and differentiable, and the derivative $q^{\prime}(x)$ is bounded and Hölder continuous with some exponent $\alpha>0$, i.e.

$$
\sup _{x \in R^{+}}\left(\sup _{y} \frac{\left|q^{\prime}(x)-q^{\prime}(y)\right|}{|x-y|^{\alpha}}\right)<C .
$$

Then the absolutely continuous part of the spectral measure $\rho_{\mathrm{ac}}^{H_{q}}$ associated with the Stark operator $\mathrm{H}_{q}$ fills the whole real axis.

Proof. The strategy of the proof will be the same as for decaying case. We begin by analyzing equations (1.4), (1.5):

$$
\begin{gathered}
(\log R)^{\prime}(\xi, \lambda)=\frac{1}{2} V(\xi) \sin (2 \theta(\xi, \lambda)), \\
\theta^{\prime}(\xi, \lambda)=1-\frac{1}{2} V(\xi)+\frac{1}{2} V(\xi) \cos (2 \theta(\xi, \lambda)) .
\end{gathered}
$$

We recall that

$$
V(\xi)=\frac{5}{36 \xi^{2}}-\frac{\lambda-q\left(c \xi^{\frac{2}{3}}\right)}{c \xi^{\frac{2}{3}}} .
$$

To treat the phase equation, we introduce quantities similar to $\gamma$ and $\sigma$ in the previous proof:

$$
\begin{gathered}
\tilde{\gamma}(\xi, \lambda)=2 \xi-\int_{0}^{\xi} V(\eta) d \eta \\
\tilde{\sigma}(\xi, \lambda)=2 \theta(\xi, \lambda)-\tilde{\gamma}(\xi, \lambda) .
\end{gathered}
$$

The computation completely repeating that in the proof of Theorem 1.1 allows us to conclude that $R(\xi, \lambda)$ is bounded for a.e. $\lambda$ if the expression

$$
V(\xi, \lambda)\left[\int_{\xi}^{\infty} V(\eta, \lambda) \exp \left( \pm\left(2 i \eta-i \int_{0}^{\eta} V(t, \lambda) d t\right)\right) d \eta\right]^{2}
$$

is well-defined and absolutely integrable for a.e. $\lambda$. Let us now consider this control expression more carefully. Consider the integral 


$$
\begin{gathered}
w_{ \pm}(\xi, \lambda)=\int_{\xi}^{\infty} V(\eta, \lambda) \exp \left( \pm\left(2 i \eta-\int_{0}^{\eta} V(t, \lambda) d t\right)\right) d \eta \\
=\int_{\xi}^{\infty} \frac{q\left(c \eta^{\frac{2}{3}}\right)}{c \eta^{\frac{2}{3}}} \exp \left( \pm\left(2 i \eta-\int_{0}^{\eta} V(t, \lambda) d t\right)\right) d \eta \\
+\int_{\xi}^{\infty}\left(\frac{5}{36 \eta^{2}}-\frac{\lambda}{c \eta^{\frac{2}{3}}}\right) \exp \left( \pm\left(2 i \eta-\int_{0}^{\eta} V(t, \lambda) d t\right)\right) d \eta .
\end{gathered}
$$

We see that the second integral on the right-hand side converges for all $\lambda$ and is $O\left(\xi^{-\frac{1}{3}}\right)$ by Lemma 1.3. Hence it remains to study the first integral. We rewrite this integral as follows:

$$
\int_{\xi}^{\infty} q\left(c \eta^{\frac{2}{3}}\right) \exp \left(\mp i\left(-\frac{5}{36 \eta}+\int_{0}^{\eta} q\left(c t^{\frac{2}{3}}\right) c^{-1} t^{-\frac{2}{3}} d t\right)\right) c^{-1} \eta^{-\frac{2}{3}} \exp \left( \pm\left(2 i \eta+3 i \lambda c^{-1} \eta^{\frac{1}{3}}\right)\right) d \eta .
$$

Let us introduce a short-hand notation $g(\eta)$ for the function

$$
\exp \left( \pm i\left(-\frac{5}{36 \eta}+\int_{0}^{\eta} q\left(c t^{\frac{2}{3}}\right) c^{-1} t^{-\frac{2}{3}} d t\right)\right)
$$

Note that $g$ is continuously differentiable and $|g(\eta)|+\left|g^{\prime}(\eta)\right|$ is bounded. In (2.2) we differentiate by parts, differentiating the first two terms and integrating the next two. Note that

$$
\int_{\xi}^{\infty} \eta^{-\frac{2}{3}} \exp \left( \pm\left(2 i \eta+3 i \lambda c^{-1} \eta^{\frac{1}{3}}\right)\right) d \eta= \pm \frac{1}{2 i} \xi^{-\frac{2}{3}} \exp \left( \pm 2 i \xi \pm 3 i \lambda c^{-1} \xi^{\frac{1}{3}}\right)+O\left(\xi^{-\frac{4}{3}}\right) .
$$

Given this observation, we obtain that the integral (2.2) is equal to

$$
\begin{array}{r}
O\left(\xi^{-\frac{2}{3}}\right) \pm\left(\frac{c^{-1}}{6 i} \int_{\xi}^{\infty}\left(2 c \eta^{-\frac{1}{3}} q^{\prime}\left(c \eta^{\frac{2}{3}}\right) \mp i q^{2}\left(c \eta^{\frac{2}{3}}\right) c^{-1} \eta^{-\frac{2}{3}} \mp i \frac{5 q\left(c \eta^{\frac{2}{3}}\right)}{36 \eta}\right)\right. \\
\left.\times g(\eta) \exp \left( \pm 2 i \eta \pm 3 c^{-1} i \lambda \eta^{\frac{1}{3}}\right) \eta^{-\frac{2}{3}}\left(1+O\left(\eta^{-\frac{2}{3}}\right)\right)\right) \\
=O\left(\xi^{-\frac{1}{3}}\right) \pm \frac{c^{-1}}{6 i} \int_{\xi}^{\infty} q^{\prime}\left(c \eta^{\frac{2}{3}}\right) \eta^{-1} g(\eta) \exp \left( \pm 2 i \eta \pm 3 c^{-1} i \lambda \eta^{\frac{1}{3}}\right) d \eta
\end{array}
$$

By assumption, $q^{\prime}$ is Hölder continuous uniformly over $R$ with exponent $\alpha$. Hence for every $h>0$,

$$
q^{\prime}\left(c(\eta+h)^{\frac{2}{3}}\right)-q^{\prime}\left(c \eta^{\frac{2}{3}}\right)=C(\eta, h) \eta^{-\frac{\alpha}{3}} h^{\alpha},
$$

where $C(\eta, h)$ is bounded uniformly for all $\eta, 0<h<1$. In the rest of the proof we will keep the notation $C(\eta, h)$ for different functions satisfying the same property. 
Let us integrate by parts the last integral " $\alpha$ " times. That is, let $h>0$ be fixed, such that $\exp ( \pm 2 i h)-1 \neq 0$. Then

$$
\begin{gathered}
\int_{\xi}^{\infty} q^{\prime}\left(\eta^{\frac{2}{3}}\right) g(\eta) \eta^{-1} \exp \left( \pm 3 c^{-1} i \lambda \eta^{\frac{1}{3}}\right) \exp ( \pm 2 i \eta)(\exp ( \pm 2 i h)-1) d \eta \\
=O\left(\xi^{-1}\right)+\int_{\xi}^{\infty}\left(q^{\prime}\left((\eta+h)^{\frac{2}{3}}\right)(\eta+h)^{-1} g(\eta+h) \exp \left( \pm 3 c^{-1} i \lambda(\eta+h)^{\frac{1}{3}}\right)\right. \\
\left.-q^{\prime}\left(\eta^{\frac{2}{3}}\right) \eta^{-1} g(\eta) \exp \left( \pm 3 c^{-1} i \lambda \eta^{\frac{1}{3}}\right)\right) \exp ( \pm 2 i \eta) d \eta .
\end{gathered}
$$

Note that

$$
\exp \left( \pm 3 c^{-1} i \lambda(\eta+h)^{\frac{1}{3}}\right)-\exp \left( \pm 3 c^{-1} i \lambda \eta^{\frac{1}{3}}\right)=\eta^{-\frac{2}{3}} C_{\lambda}(\eta, h) .
$$

Hence we can rewrite the last integral as

$O\left(\xi^{-\frac{2}{3}}\right)+\int_{\xi}^{\infty}\left(q^{\prime}\left((\eta+h)^{\frac{2}{3}}\right)(\eta+h)^{-1} g(\eta+h)-q^{\prime}\left(\eta^{\frac{2}{3}}\right) \eta^{-1} g(\eta)\right) \exp \left( \pm 2 i \eta \pm 3 c^{-1} i \lambda \eta^{\frac{1}{3}}\right) d \eta$.

Since we have

$$
\left|(\eta+h)^{-1} g(\eta+h)-\eta^{-1} g(\eta)\right| \leq C \eta^{-1},
$$

a simple computation taking into account (2.3) and (2.4) shows that the last expression is equal to

$$
O\left(\xi^{-\frac{2}{3}}\right)+\int_{\xi}^{\infty} C(\eta, h) \eta^{-1-\frac{\alpha}{3}} \exp \left( \pm 2 i \eta \pm 3 c^{-1} i \lambda \eta^{\frac{1}{3}}\right) d \eta
$$

By Corollary 1.6, we obtain that the last expression converges for a.e. $\lambda$ and is bounded by $C \xi^{-\frac{1}{3}\left(\frac{1}{2}+\alpha\right)+\epsilon}$ for every $\epsilon>0$. Therefore we have completed an estimation of the integral $w_{ \pm}(\xi, \lambda)$ and obtained that for a.e. $\lambda$

$$
w_{ \pm}(\xi, \lambda) \leq C(\lambda)\left(\xi^{-\frac{1}{3}}+\xi^{-\frac{1}{3}\left(\frac{1}{2}+\alpha\right)+\epsilon}\right) .
$$

Taking into account the fact that $|V(\xi)| \leq C \xi^{-\frac{2}{3}}$, this implies that for a.e. $\lambda$ the control expression (14) is bounded by $C(\lambda) \xi^{-1-\frac{2 \alpha}{3}+\epsilon}$ and hence is absolutely integrable. This allows us to find the asymptotic behavior of solutions of the equation (1.3) for a.e. $\lambda$. Applying Lemma 1.2, we find that for a.e. $\lambda$ there is no subordinate solution of the original equation (1.1). This completes the proof of Theorem 2.1.

\section{ACKNOWLEDGMENT}

I thank Professor Stolz for suggesting the problem and Professor Simon for stimulating discussions. I am also grateful to Professor Anne Boutet de Monvel for informing me about the related work of J. Sahbani. Research at MSRI supported in part by NSF grant DMS 9022140. 


\section{REFERENCES}

[1] J. Asch, P. Duclos and P. Exner, Stark-Wannier Hamiltonians with pure point spectrum, in Differential Equations, Asymptotic Analysis, and Mathematical Physics (Potsdam, 1996), Akademie Verlag, Berlin, 1997. MR 98f:81066

[2] J. Avron and B. Simon, Transient and recurrent spectrum, J. Funct. Anal. 43(1981), 1-31. MR 83c: 47008

[3] J.E. Avron, P. Exner and Y. Last, Periodic Schrödinger operators with large gaps and Wannier-Stark ladders, Phys. Rev. Lett. 72(1994), 896-899.

[4] F. Bentosela et al., Schrödinger operators with an electric field and random or deterministic potentials, Commun. Math. Phys. 88(1983), 387-397. MR 85f:34036

[5] P. Exner, The absence of the absolutely continuous spectrum for $\delta^{\prime}$ Wannier-Stark ladders, J. Math. Phys. 36(9) (1995), 4561-4570. MR 96g:81050

[6] D.J. Gilbert, On subordinacy and analysis of the spectrum of Schrödinger operators with two singular endpoints, Proc. Roy. Soc. Edinburgh Sect. A 112(1989), 213-229. MR 90j:47061

[7] D.J. Gilbert and D.B. Pearson, On subordinacy and analysis of the spectrum of onedimensional Schrödinger operators, J. Math. Anal. Appl. 128(1987), 30-56. MR 89a:34033

[8] P. Hartman, Ordinary Differential Equations, Birkhäuser, Boston 1982. MR 83e:34002

[9] A. Kiselev, Absolutely continuous spectrum of one-dimensional Schrödinger operators and Jacobi matrices with slowly decaying potentials, Commun. Math. Phys.179(1996), 377-400. MR 97e:81027

[10] A. Kiselev, Stability of the absolutely continuous spectrum of Schrödinger equation under perturbations by slowly decreasing potentials and a.e. convergence of integral operators, Duke Math. J. 94(1998), 619-646. CMP 98:17

[11] A. Kiselev and B. Simon, Rank one perturbations in infinitesmal coupling, J. Funct. Anal. 130(1)(1995), 345-356. MR 96e:47012

[12] Y. Last, Quantum dynamics and decompositions of singular continuous spectra, J. Funct. Anal. 142(2)(1996), 406-445. MR 97k:81044

[13] S.N. Naboko, Dense point spectra of Dirac and Schrödinger operators, Theor. Math. 68(1986), 18-28. MR 88h:81029

[14] S.N. Naboko and A.B. Pushnitskii, Point spectrum on a continuous spectrum for weakly perturbed Stark type operators, Funct. Anal. Appl. 29(4)(1995), 248-257. MR 96k:34187

[15] J. von Neumann and E.P. Wigner, Über merkwürdige diskrete eigenwerte, Z. Phys. 30(1929), $465-467$.

[16] W. Rudin, Real and Complex Analysis, New York, McGraw-Hill, 1987. MR 88k:00002

[17] J. Sahbani, Propagation theorems for some classes of pseudodifferential operators, J. Math. Anal. Appl. 211 (1997), 481-497. MR 98e:47078

[18] B. Simon, Spectral analysis of rank one perturbations and applications, Proc. 1993 Vancouver Summer School in Mathematical Physics.

[19] B. Simon, Bounded eigenfunctions and absolutely continuous specta for one-dimensional Schrödinger operators, Proc. Amer. Math. Soc. 124(11), 3361-3369. MR 97a:34223

[20] G. Stolz, Bounded solutions and absolute continuity of Sturm-Liouville operators, J. Math. Anal. Appl. 169(1992), 210-228. MR 93f:34141

[21] J. Walter, Absolute continuity of the essential spectrum of $-d^{2} / d x^{2}+q(x)$ without monotony of $q$, Math. Z. 129(1)(1972), 83-94. MR 48:4399

[22] A. Zygmund, A remark on Fourier transforms, Proc. Camb. Phil. Soc. 32, 321-327 (1936).

Mathematical Sciences Research Institute, 1000 Centennial Drive, Berkeley, CaliFORNIA 94720

Current address: Department of Mathematics, University of Chicago, 5734 S. University Avenue, Chicago, Illinois 60637

E-mail address: kiselev@math.uchicago.edu 